\title{
Conocimientos, actitudes y prácticas sobre la prueba de Papanicolaou
}

\author{
Fernando Bazán ${ }^{1}$, Margarita Posso ${ }^{1}$, César Gutiérrez ${ }^{2}$
}

Resumen

Palabras clave

\begin{abstract}
Objetivos: Determinar los conocimientos, actitudes y prácticas con respecto a la prueba de Papanicolaou (Pap); hallar la asociación entre la edad y grado de instrucción frente a dichas variables y describir las características sobre la práctica periódica del Pap. Diseño: Analítico y transversal. Lugar: Hospital Nacional Docente Madre-Niño 'San Bartolomé'. Pacientes: Mujeres en edad fértil de consultorios externos. Intervenciones: Cuestionario estructurado, autodesarrollado, durante noviembre-2005 a enero-2006. Para el análisis estadístico se utilizó: X², t student, Anova y OR. Principales medidas de resultados: Nivel de conocimientos, actitudes y prácticas en mujeres sobre el Pap, y asociaciones y características sobre su práctica periódica. Resultados: De 501 encuestadas, en 63\% se encontró nivel bajo de conocimiento, en $66 \%$ una actitud desfavorable y en $71 \%$ una práctica incorrecta sobre la prueba de Papanicolaou; se obtuvo un $O R=1,45$ (IC = 0,98 a 2,16) entre el nivel de conocimiento y la práctica correcta; la difusión por los medios de comunicación sobre el Pap fue 6,2\% y la mayor barrera para no tener una práctica periódica del Pap fue el género masculino del ejecutor (34,2\%). Conclusiones: Los niveles de conocimiento, actitudes y prácticas, en nuestra población, son bajos. El mayor nivel de conocimiento no está relacionado con la práctica correcta del Pap. Existe poca difusión sobre el Pap por los medios de comunicación y las limitantes a que las mujeres se tomen el Pap son principalmente psicológicas.
\end{abstract}

Neoplasias del cuello uterino; conocimientos, actitudes y prácticas; carcinoma de células escamosas; medicina preventiva.
Knowledge, attitudes and practices on Papanicolaou cervical smear

\section{Abstract}

Objetives: To determine the level of knowledge, attitudes and practices with respect to the Papanicolaou cervical smear test (Pap), to find association between age and learning level, and to describe the characteristics on Pap's periodic practice. Design: Analytical and cross-sectional study. Setting: San Bartolome Mother and Child National Teaching Hospital. Patients: Women in fertile age. Interventions: Structured questionnaire, self-developed, from November 2005 through January 2006. $\mathrm{X}^{2}$, t student, Anova and OR were used for statistical analysis. Main outcome measures: Level of knowledge, attitudes and practices on Papanicolaou smear, as well as associations and characteristics on its periodical practice. Results: Of 501 surveys, 63\% showed low level of knowledge, $66 \%$ had an unfavorable attitude, and $71 \%$ an incorrect practice of Pap smear; OR between knowledge level

Estudiantes de la Facultad de Medicina Humana, UNMSM. Lima, Perú. Instituto de Medicina Tropical Daniel A. Carrión, Facultad de Medicina Humana, UNMSM. Lima, Perú. and correct practice was 1,45 (IC = 0,98-2,16). Mass media information on Pap smear was 6,2\% and the greater barrier to having a Pap smear periodic practice was the performer's gender (masculine) (34,2\%). Conclusions: Level ofknowledge, attitudes and practices in our population are low. The greater level of Pap knowledge is notrelated to correct practice. There is poor diffusion on Pap smear by the mass media. Observations on not having a Pap periodic practice are mainly psychological.

Key words: Cervix neoplasms; knowledge, attitudes, practice; carcinoma, squamous cell; preventive medicine.

\section{INTRODUCCIÓN}

El carcinoma de células escamosas de cérvix es el segundo cáncer más frecuente de la población femenina en el mundo $\left({ }^{1}\right)$, siendo una neoplasia prevenible cuando su diagnóstico es oportuno y cuando se realiza un adecuado tratamiento de las lesiones premalignas. Debido a su desarrollo en un período largo de tiempo, puede ser detecta- 
do con una muestra citológica exfoliativa de cuello uterino (Papanicolaou, Pap), antes de que la mujer experimente síntomas $\left({ }^{2}\right)$.

América del Sur tiene una de las tasas de incidencia (30,9 x 100000 mujeres) y mortalidad $(11,97$ x 100000 mujeres $)$ de cáncer de cérvix más altas del mundo $\left({ }^{3}\right)$. De acuerdo a la estadística del Instituto Nacional de Enfermedades Neoplásicas - INEN del Perú (1994-1997), el cáncer de cérvix es el segundo cáncer ginecológico más frecuente en Lima Metropolitana $\left({ }^{4}\right)$. La tasa de mortalidad en el período 1994 - 1997, en Lima, fue $10,94 \times 100000$ mujeres $\left({ }^{4}\right)$, aumentando a $15,8 \times 100000$ mujeres para el año $2000\left({ }^{5}\right)$. Tendencia similar se observa para la incidencia, que de una tasa de $23,5 \times 100000$, en $1994-1997\left(^{4}\right)$, pasó a 39,9 , en el año $2000\left(^{5}\right)$.

La prueba del Pap ha sido utilizada para el diagnóstico oportuno de lesiones precursoras, y ha significado un gran avance en la prevención del cáncer de cérvix $\left(^{6}\right)$, pues se ha demostrado que su utilización periódica reduce de manera importante la morbilidad y mortalidad por esta neoplasia $\left({ }^{7-9}\right)$. A largo plazo, el tamizaje y tratamiento de lesiones premalignas tienen un menor costo y mayor beneficio comparado con el tratamiento médico-quirúrgico de los carcinomas de cérvix $\left({ }^{10}\right)$.

Una amplia cobertura y elevados estándares de calidad en los programas de detección son factores importantes para que este cáncer haya disminuido significativamente $(90 \%)$ en los países Nórdicos, Canadá y EE UU $\left.{ }^{(11,12}\right)$. Por el contrario, esta situación no se ha observado en países en desarrollo; tal es el caso de México, donde, en 1974, se implementó el Programa Nacional de Detección Oportuna de Cáncer (PNDOC) y a pesar de ello la tasa de mortalidad por cáncer de cérvix entre 1974 y 1999 no ha disminuido, debido a su baja cobertura y sus bajos estándares de calidad $\left({ }^{13}\right)$.
La última Guía de la Sociedad Americana de Cáncer para la detección temprana de Neoplasia de Cérvix y Cáncer, publicada en 2002, recomienda que el intervalo de tamizaje se realice tres años después del inicio de las relaciones sexuales vaginales (o después de los 21 años de edad), continuándose anualmente hasta los 30 años, edad en la cual, al tener 3 o más pruebas del Pap técnicamente satisfactorias, informadas como normales o citología negativa, el tamizaje puede ser realizado cada 2 o 3 años, hasta la edad de 70 años $\left({ }^{14}\right)$.

Estudios muestran que un mayor porcentaje de participación (prácticas) estarían relacionados a un mayor grado de conocimientos y actitudes de la participante $\left({ }^{15-16}\right)$. Niveles de educación y niveles económicos altos y actitudes positivas frente al Pap han demostrado un mayor uso de esta prueba $\left({ }^{17}\right)$. Es por eso importante investigar las prácticas, conocimientos y actitudes de las mujeres con relación a la prueba del Pap.

En nuestro país, el cáncer de cérvix representa una patología de alta incidencia y alta mortalidad, pero no se ha encontrado estudios previos que evalúen los conocimientos, las actitudes y las prácticas sobre el Pap ni que describan características sobre la práctica periódica de la misma. Por lo que, los resultados obtenidos pueden coadyuvar a replantear estrategias de intervención oportunas que permitan incrementar la tasa de cobertura en las mujeres que asisten a los servicios de salud, a fin de disminuir la incidencia, mortalidad y costos de atención de los tratamientos para cáncer de cérvix en el Perú.

Los objetivos del trabajo fueron determinar los conocimientos, actitudes y prácticas con respecto al Pap, determinar si existe asociación entre nivel de conocimiento, prácticas y actitudes frente a la edad y el grado de instrucción, y describir las características sobre la práctica periódica del Papanicolaou. 


\section{MATERIALES Y MÉTODOS}

El estudio fue de tipo analítico, de corte transversal, mediante una selección no aleatoria en la población de mujeres atendidas en los tres consultorios externos de Ginecología del Hospital Nacional Docente Madre - Niño 'San Bartolomé', durante el período noviembre 2005 a enero 2006. Se utilizó la fórmula para el cálculo del tamaño muestral (501 pacientes mujeres), que proporcionó el programa Epi-Info 2002, basándose en los criterios de nivel de confianza de $95 \%$, un error relativo de $5 \%$, $p=25 \%, \mathrm{q}=75 \%$.

A cada mujer se le aplicó un cuestionario estructurado, auto-desarrollado, anónimo, previo consentimiento informado verbal, con 9 preguntas cerradas y 6 preguntas abiertas; constituido en 5 bloques: a) Datos personales: edad y grado de instrucción; b) Prácticas sobre la prueba del Pap; c) Conocimientos sobre la prueba del Pap; d) Actitudes sobre la prueba del Pap; y, e) Características sobre la práctica periódica del Pap.

El cuestionario fue elaborado por los autores, después de llevar a cabo una búsqueda de estudios similares. Se realizó un piloto en 30 pacientes del mismo nosocomio, que permitió corregir el número, la claridad, la dificultad, el orden, el tiempo de duración de las preguntas y formato del cuestionario. Posteriormente, fue sometido a juicio de expertos, constituido por dos médicos gineco-obstetras y un médico de cirugía gineco-oncológica del hospital referido.

Los datos recolectados fueron tabulados y analizados en el programa SPSS 11,0, trabajándose con un nivel de significancia de $95 \%$. Se realizó en primer lugar un análisis descriptivo para cada una de las cinco áreas que formaron el cuestionario. La calificación de los niveles de conocimientos, actitudes y prácticas se realizó por criterio de los autores, en base a búsqueda bibliográfica y consultoría especializada (Tabla 1).
Se empleó luego la prueba chi cuadrado $\left(X^{2}\right)$, $\mathrm{T}$ de student $(t)$ y Anova $(f)$, para determinar si existe asociación entre conocimientos, actitudes y prácticas frente a edad y grado de instrucción; y se halló la fuerza de asociación entre las principales variables, mediante el cálculo del OR.

\section{RESULTADOS}

De un total de 501 pacientes encuestadas, $42,7 \%$ (214) fue menor de 30 años y 58,3\% (287) mayor de 30 años. Según el grado de instrucción, 8,9\% (45) correspondió a ninguno y primaria, $53,3 \%$ (267) a secundaria y $37,8 \%(189)$ a superior.

Con respecto a los conocimientos, a la pregunta de dónde se toma la muestra para el Pap, 48,9\% respondió correctamente; el mayor error, en $37,3 \%$, fue creer que la muestra proviene de la secreción vaginal. Sobre el propósito del Pap, el 54,3\% de mujeres respondió incorrectamente, siendo el principal error (37\%) responder que el Pap detecta cualquier cáncer ginecológico. Frente a la frecuencia de toma del Pap, 69\% del grupo menor de 30 años respondió correctamente, contrastando con solo 4,9\% del grupo mayor de 30 años (Tabla 2). Con estos resultados, se observó que solo 10,6\% tiene conocimientos altos sobre el Pap, mientras que $89,4 \%$ de la población estudiada presentó conocimientos bajos e intermedios (Figura 1).

Con respecto a las actitudes, tan solo $34 \%$ de la población refirió sentirse dispuesta cuando se le solicitaba realizarse un Pap, mientras que $30 \%$ se refirió preocupada, $16,2 \%$ incómoda y $10,8 \%$ avergonzada. A la pregunta si es que creen que es importante hacerse el Pap periódicamente, 98,8\% respondió afirmativamente; $98,4 \%$ respondió tener interés por conocer más sobre la prueba de Pap. Con estos resultados, se observó que $66,1 \%$ de la población ostentaba una actitud desfavorable con respecto al Pap (Figura 1). 
Fernando Bazán y col.

Tabla 1. Calificación de los niveles de conocimientos, actitudes y prácticas.

\begin{tabular}{|c|c|c|}
\hline Variable & Calificación & Respuestas \\
\hline \multirow[t]{3}{*}{ Conocimientos } & Nivel alto & $\begin{array}{l}\text { Si respondió correctamente a las } 3 \text { preguntas sobre el conocimiento: } \\
\text { a) Lugar de toma de muestra: del cuello del útero (cérvix). } \\
\text { b) Propósito: detección temprana del cáncer de cuello uterino. } \\
\text { c) Frecuencia en que debería tomarse un Pap: } \\
\text { una vez al año, si su edad < } 30 \text { años o } \\
1 \text { vez cada } 2 \text { a } 3 \text { años, si su edad } \geq 30 \text { años }\end{array}$ \\
\hline & Nivel intermedio & 2 respuestas correctas sobre conocimiento. \\
\hline & Nivel bajo & $\leq 1$ respuesta correcta. \\
\hline \multirow[t]{2}{*}{ Actitudes } & Favorable & $\begin{array}{l}\text { Si respondió favorablemente a las } 3 \text { respuestas sobre actitudes: } \\
\text { a) Conducta frente a petición de realización de Pap: estar dispuesta. } \\
\text { b) Cree que es importante realizarse periódicamente del Pap: Sí. } \\
\text { c) Tenga interés por adquirir más conocimientos sobre Pap: Sí. }\end{array}$ \\
\hline & Desfavorable & $\leq 2$ respuestas favorables de actitudes, de 3 preguntas de actitudes. \\
\hline \multirow[t]{2}{*}{ Prácticas } & Correcta & $\begin{array}{l}\text { Si respondió correctamente a } 2 \text { o más de las } 3 \text { preguntas sobre prácticas: } \\
\text { a) Frecuencia con la que se realiza un Pap: } \\
1 \text { vez al año, si su edad < } 30 \text { años o } \\
1 \text { vez cada } 2 \text { a } 3 \text { años, si su edad } \geq 30 \text { años } \\
\text { b) Motivo: por indicación médica o por iniciativa propia. } \\
\text { c) Lugar de realización de sus Pap: hospital, posta o consultorio particular } \\
\text { - Siendo necesaria que a) sea una de las } 3 \text { respuestas correctas. }\end{array}$ \\
\hline & Incorrecta & $\leq 1$ respuesta correcta. \\
\hline
\end{tabular}

Con respecto a las prácticas, a la pregunta sobre la frecuencia en que las encuestadas se realizan una prueba de Pap, $63,3 \%$ las realizaba con una frecuencia adecuada, mientras que $16 \%$ solo se había realizado un Pap en toda su vida y $11 \%$ nunca se la había realizado. El principal motivo para realizarse un Pap fue iniciativa propia, $53,2 \%$, mientras $39,8 \%$ fue por solicitud médica. El $67,8 \%$ refirió al Hospital

Tabla 2. Frecuencia en que las mujeres manifestaron deberían realizarse un Pap.

\begin{tabular}{lrlrl}
\hline ¿Cada cuánto tiempo debe & \multicolumn{4}{c}{ Grupo de edad } \\
\cline { 2 - 5 } Ud. realizarse un Pap? & \multicolumn{3}{c}{$<30$ años } & \multicolumn{2}{c}{$>$ 30 años } \\
\hline Desconozco & $21,6 \%$ & $(46)$ & $12,2 \%$ & $(35)$ \\
1 vez al año & $\mathbf{6 9 , 0 \%}$ & $\mathbf{( 1 4 7 )}$ & $76,7 \%$ & $(220)$ \\
3 veces al año & $6,6 \%$ & $(14)$ & $6,3 \%$ & $(18)$ \\
1 vez cada 2 o 3 años & $2,8 \%$ & $(7)$ & $\mathbf{4 , 9 \%}$ & $\mathbf{( 1 4 )}$ \\
Total & $100,0 \%$ & $(214)$ & $100,0 \%$ & $(287)$ \\
\hline
\end{tabular}

como el lugar más concurrido para realización del Pap, mientras que $25,3 \%$ indicó a la posta o centro de salud y $6,9 \%$ a un consultorio particular. Con estos resultados, se observó que $71,3 \%$ de mujeres poseía prácticas incorrectas sobre el Pap (Figura 1).

Se halló que existe asociación estadísticamente significativa entre grado de instrucción y conocimientos; es decir, a mayor grado de instrucción, mayor conocimiento $\left(X^{2}=19,6 ; p=0,001\right)$, así como asociación estadísticamente significativa entre grado de instrucción y actitudes, es decir, a mayor grado de instrucción se observó actitudes más favorables $\left(X^{2}=8,2\right.$; $p=0,016)$. Pero, si bien las pacientes con grado de instrucción superior presentan prácticas correctas en una mayor proporción, no se halló una asociación estadísticamente significativa entre grado de instrucción y prácticas $\left(X^{2}=5,7 ; p=0,057\right)$ 


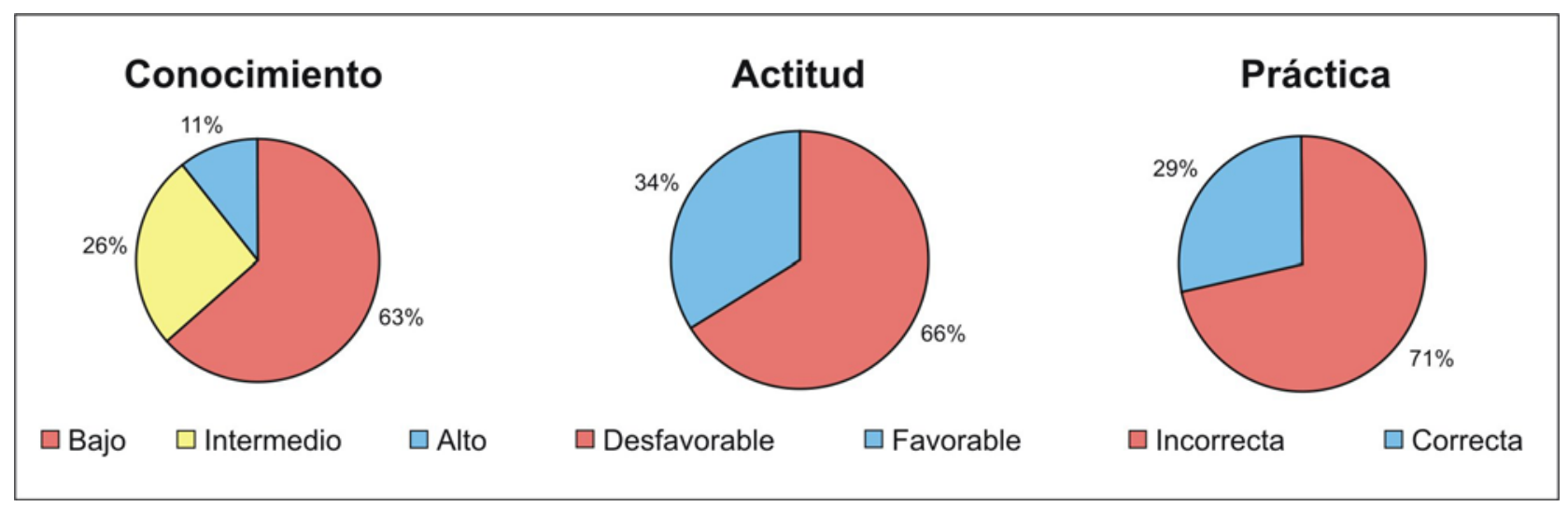

Figura 1. Grado de conocimiento, actitud y práctica sobre la prueba de Pap en la población estudiada.

Las mujeres con ningún grado de instrucción o solo primaria tuvieron 4,12 veces la probabilidad de tener conocimientos bajos en comparación a las mujeres con grado de instrucción superior $(\mathrm{OR}=4,12 ; \mathrm{IC}=1,72$ a $10,16)$. Las mujeres con grado de instrucción secundaria tuvieron 1,84 veces la probabilidad de tener conocimientos bajos en comparación a las mujeres con grado de instrucción superior $(\mathrm{OR}=1,84 ; \mathrm{IC}=1,23$ a $2,75)$. Las mujeres con ningún grado de instrucción o solo primaria tuvieron 3,04 veces la probabilidad de tener actitudes desfavorables en comparación a las mujeres con grado de instrucción superior $(\mathrm{OR}=3,04$; $\mathrm{IC}=1,27$ a 7,52$)$. No se observó diferencia significativa entre el grado de instrucción secundaria y superior, con respecto a las actitudes. Las mujeres con grado de instrucción secundaria tuvieron 1,63 veces la probabilidad de tener prácticas incorrectas en comparación a las mujeres con grado de instrucción superior $(\mathrm{OR}=1,63 ; \mathrm{IC}=1,06$ a $2,51)$. No se observó diferencia entre el grado de instrucción ninguno-primaria y superior, con respecto a las prácticas (Tabla 3 ).

Al comparar el promedio de edad y las prácticas (correcta o incorrecta) del Pap, no se halló diferencia significativa; 33,1 años entre las pacientes con prácticas correctas y 33 años entre las pacientes con prácticas incorrectas $(t=0,05 ; p=0,956) \mathrm{Al}$ comparar el promedio de edad y las actitudes (favorable o desfavorable) del Pap, no se halló diferencia significativa: 33,2 años entre las pacientes con actitud favorable y 32,9 años entre las pacientes con actitud desfavorable $(t=-0,39 ; p=0,690)$. Al comparar el promedio de edad y los conocimientos (nivel alto $=26,5$ años, intermedio $=33$ años y bajo $=34,1$ años) del Pap, se halló diferencia estadísticamente significativa $(f=15,5 ; p<0,001)$, es decir, a menor edad mayor conocimiento.

Las mujeres menores de 30 años mostraban 2,4 veces la probabilidad de tener conocimientos altos o intermedios en comparación a las mujeres de 30 años o más $(\mathrm{OR}=2,4 ; \mathrm{IC}=1,65$ a 3,47$)$. No se halló relación entre las mujeres menores de 30 años y las mayores de 30 años, con respecto a actitudes ( $\mathrm{OR}=0,99 ; \mathrm{IC}=0,68$ a 1,44$)$. Las mujeres menores de 30 años tuvieron 1,6 veces la probabilidad de tener prácticas correctas en comparación a las mujeres de 30 o más años (IC=1,08 a 2,35) (Tabla 4).

No existió relación estadísticamente significativa entre los conocimientos y las prácticas. ( $\mathrm{OR}=1,456$; $\mathrm{IC}=0,98$ a 2,16$) \mathrm{ni}$ relación entre las actitudes y las prácticas $(\mathrm{OR}=1,30 ; \mathrm{IC}=0,869$ a 1,95$)$.

Con respecto a las características sobre la práctica periódica del Pap, se encontró 
Fernando Bazán y col.

Tabla 3. Asociación entre grado de instrucción con conocimientos, actitudes y prácticas.

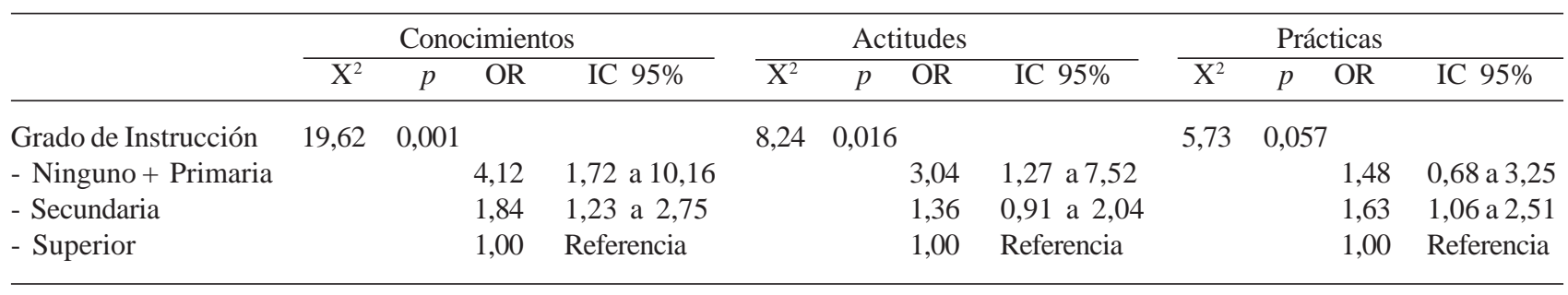

que los principales motivos por los cuales no se realizaban el Pap fueron que $34,2 \%$ preferiría que el médico fuera mujer, $24 \%$ refirió falta de tiempo y $22 \%$ miedo a que el resultado no fuera normal. Sobre la fuente de información del Pap, tan solo $8,4 \%$ fue mediante campañas de salud, mientras que $34,7 \%$ la obtuvo en su hospital, $18,8 \%$ mediante una amistad o familiar, $15,8 \%$ en su centro de salud y $6,2 \%$ a través de medios de comunicación. El 69,6\% refirió que el lugar donde se sentía más cómoda para hacerse el Pap era el hospital.

\section{DISCUSIÓN}

En nuestro estudio hallamos que solo $10,6 \%$ tenía conocimientos altos sobre el Pap, mientras que $89,4 \%$ de la población estudiada presentó conocimientos bajos e intermedios; resultado comparable con el $75,5 \%$ de conocimiento bajo encontrado por Baradaran $\left({ }^{18}\right)$, en una población iraní. A la pregunta sobre el propósito del Pap, un alarmante $54,3 \%$ de mujeres respondió incorrectamente, cifra similar al $49 \%$ encontrada por Giganchi $\left({ }^{19}\right)$, en una población de Kenia, y $62 \%$ encontrado por Idestrom $\left({ }^{15}\right)$ en una población suiza.
Hallamos una clara actitud desfavorable $(66,1 \%)$ frente a la toma del Pap: $57 \%$ refirió sentirse incómoda, avergonzada o preocupada, cuando se le solicitaba esta prueba, resultado comparable con el $65 \%$ encontrado por Idestrom $\left({ }^{15}\right)$.

Se evidenció prácticas incorrectas en $71,3 \%$ de la población. El 16\% se había realizado solamente un Pap en toda su vida, comparable con el $22 \%$ hallado por Giganchi $\left({ }^{19}\right)$, en una población de Kenia, y haciendo gran contraste con el $94,2 \%$ hallado por Klug $\left({ }^{20}\right)$, en una población alemana. Además, $11 \%$ de nuestra población nunca se había realizado el Pap, ubicándonos en un punto intermedio entre el $78 \%$ hallado por Giganchi $\left({ }^{19}\right)$ y un $0,75 \%$ hallado por Klug $\left({ }^{20}\right)$. Se demuestra así nuestra aún baja cobertura de la prueba, en comparación a países desarrollados.

Se halló asociación estadísticamente significativa entre el grado de instrucción con los conocimientos y las actitudes; así, a mayor grado de instrucción hay mayor probabilidad de tener un mayor nivel de conocimientos y una actitud favorable; estos resultados son compatibles con los encontrados por Lazcano-Ponce $\left({ }^{21}\right)$ en una población en México.

Tabla 4. Asociación entre edad con conocimientos, actitudes y prácticas.

\begin{tabular}{|c|c|c|c|c|c|c|c|c|c|c|c|c|}
\hline & \multicolumn{4}{|c|}{ Conocimientos } & \multicolumn{4}{|c|}{ Actitudes } & \multicolumn{4}{|c|}{ Prácticas } \\
\hline & $t$ & $p$ & OR & IC $95 \%$ & $t$ & $p$ & OR & IC $95 \%$ & $t$ & $p$ & OR & IC $95 \%$ \\
\hline Promedio de edad & 0,055 & 0,956 & & & 0,39 & 0,69 & & & 15,43 & $<0,001$ & & \\
\hline$<30$ años / > 30 años & & & 2,40 & 1,65 a 3,47 & & & 0,99 & 0,68 a 1,44 & & & 1,60 & 1,08 a 2,35 \\
\hline
\end{tabular}


Se halló que no existe diferencia estadísticamente significativa entre los promedios de edad y prácticas; pero, al tener menor edad $(<30$ años), es mayor la probabilidad de tener mayor nivel de conocimiento y práctica correcta del Pap. Se halló, además, que no existe relación estadísticamente significativa entre la actitud y la práctica.

En México, Lazcano-Ponce $\left({ }^{21}\right)$ y AguilarPérez $\left({ }^{22}\right)$ encontraron que el conocimiento sobre la utilidad del Pap incrementa en 29 y 6 veces más la probabilidad de utilización de la prueba, respectivamente. En contraste, y de la misma forma que Idestrom $\left({ }^{15}\right)$ y Baradaran $\left({ }^{18}\right)$, nuestros resultados demuestran que no existe relación estadísticamente significativa entre el nivel de conocimiento y la práctica correcta; a nivel personal, los autores creemos que esto puede deberse al tipo de población (hospitalaria) que fue estudiada, ya que en los grandes nosocomios el tamizaje es periódico y sistematizado (aumentando el nivel de prácticas), pero no son los lugares de donde parta la educación sanitaria; y/o, también, a que la cobertura lograda por las campañas de promoción, prevención y tamizaje (relativamente aceptables para un país en desarrollo), no promueven la educación sobre el Pap, motivo por el que no influenciarían en el nivel de conocimiento de las mujeres.

En nuestra población, se halló que los principales motivos por los cuales las mujeres no se realizaban periódicamente el Pap fueron: preferencia porque el médico fuera mujer $(34,2 \%)$, por falta de tiempo $(24 \%)$ y por miedo a que el resultado no fuera normal (22\%); comparable los hallazgos de Idestrom $\left({ }^{15}\right)$, quien encuentra la ansiedad, inseguridad y temor a la prueba como los principales factores, todos estos asociados a la sensación de pérdida de la intimidad al ser examinadas; y corroborado en una población chilena por Valenzuela $\left({ }^{23}\right)$, donde el miedo y el pudor fueron las principales barreras psicológicas. Con estos resultados, evidenciamos que, la falta de conocimien- to sobre la prueba y el género masculino de los ejecutores de la prueba son importantes barreras a superar por las políticas de prevención en nuestra población.

En nuestro estudio, hallamos sobre la fuente de información del PAP, que tan solo el $8,4 \%$ obtuvo información a través de campañas de salud y un $6,2 \%$ a través de medios de comunicación. Se demostró así la poca difusión extra-mural que existe en nuestro entorno sobre el Pap, concordando con lo hallado por Giganchi $\left({ }^{19}\right)$, en Kenia, $y$ al contrario de lo encontrado por Klug $\left({ }^{20}\right)$, en Alemania, en donde los medios de comunicación tienen una participación de hasta $53,8 \%$ en la difusión sobre la prueba del Pap.

En conclusión, en cuanto a conocimiento, actitudes y prácticas, más de $60 \%$ de las encuestadas mostró niveles bajos. Es importante destacar que, en nuestro estudio, el mayor nivel de conocimiento no estuvo relacionado con la práctica correcta de la prueba del Pap. Entre otros hallazgos, se evidenció que a mayor grado de instrucción había más probabilidad de tener mayor nivel de conocimiento y una actitud favorable. Existe poca difusión sobre la prueba del Papanicolaou por los medios de comunicación. Y las limitantes de muchas mujeres a realizarse la prueba de Papanicolaou son principalmente psicológicas.

A la luz de estos resultados, es importante reformular las estrategias educativas para atraer a las mujeres a realizarse el Pap. Por lo que, nuestro estudio podría tener implicancia en la educación sanitaria y en la política de salud de nuestro país.

\section{AGRADECIMIENTO}

A nuestra querida Alma Mater 'San Fernando', que nos dio la oportunidad de ser médicos y poder llegar a todo lo que querramos alcanzar. A la Sociedad Científica de San Fernando, a través de la cual se logró el financiamiento de este trabajo. 


\section{FINANCIAMIENTO}

Por la Universidad Nacional Mayor de San Marcos, como premio al haber ocupado el primer lugar en Proyecto de Investigación de la XIV Jornadas Científicas Sanfernandinas Estudiantiles (Set-2005).

\section{REFERENCIAS BIBLIOGRÁFICAS}

1. Schiffman M, Brinton L, Devesa S, Fraumeni J, Joseph F. Cervical cancer. Cancer Epidemiology and Prevention. New York: Oxford University Press; 2001.

2. Mohan S. Cervical screening in England and Wales: an update. Curr Opin Obstet Gynecol. 2004;16(6):491-6.

3. Parkin D, Bray F, Ferlay J, Pisani P. Global cancer statistics, 2002. CA Cancer J Clin. 2005;55:74-108.

4. Centro de Investigación Maes Heller, Instituto de Enfermedades Neoplásicas. Registro de Cáncer en Lima Metropolitana 1994 -1997. Lima: Instituto de Enfermedades Neoplásicas; 2004.

5. Parkin D, Bray F, Ferlay J, Pisani P. Estimating the world cancer burden: Globocan 2000. Int J Cancer. 2001;15(2): 153-6.

6. Fraser A, Hellmann S, Leibovici L, Levavi H. Screening for cervical cancer -an evidence- based approach. Eur J Gynaecol Oncol. 2005;26(4):372-5.

7. Quinn M, Babb P, Jones J, Allen E. Effect of screening on incidence of and mortality from cancer of cervix in England: evaluation based on routinely collected statistics. BMJ. 1999;318:904-8.

8. Sasieni P, Adams J. Effect of screening on cervical cancer mortality in England and Wales: analysis of trends with an age period cohort model. BMJ. 1999;318:1244-5.

9. Harokopos V, McDermott R. Cervical cancer screening: benefits and barriers. J Health Educ. 1996;27:351-6.

10. Jamison D, Mosley W, Measham A, Bobadilla J. Disease control priorities in developing countries. World Bank Report 12384. New York: Oxford; 1993.

11. Hristova L, Hakama M. Effect of screening for cancer in the Nordic countries on deaths, cost and quality of life up to the year 2017. Acta Oncol. 1997;36(9):1-60.

12. Bergstrom R, Sparen $P$, Adami HO. Trends in cancer of the cervix uteri in Sweden following cytological screening. Br J Cancer. 1999;81:159-66.

13. Lazcano P, Moss S, Alonso de Ruiz P, Salmerón C, Hernández A. Cervical cancer screening in developing countries: why is it ineffective? The case of Mexico. Arch Med Res.1999;30:240-50.
14. Saslow D, Runowicz C, Solomon D, Moscicki A, Smith R, Eyre H, Cohen C. American Cancer Society Guideline for the Early Detection of Cervical Neoplasia and Cancer. CA Cancer J Clin. 2002;52:342-62.

15. Idestrom M, Milson I, Andersson-Ellstrom A. Knowledge and attitudes about Pap-smear screening program: a population-based study of women aged $20-59$ years. Acta Obstet Gynecol Scand. 2002;81:962-7.

16. Fylan F. Screening for cervical cancer. a review of women's attitudes, knowledge and behaviour. Br J Gen Pract. 1998;48:1509-14.

17. Arevian M. A survey of knowledge, attitudes, and practice of cervical screening among Lebanese/Armenian women. Nursing Outlook. 1997;45(1):16-22.

18. Baradaran R, Seydi S, Mohammad A. Effects of 2 educational methods on the knowledge, attitude, and practice of women high school teachers in prevention of cervical cancer. Cancer Nursing. 2004;27(5):364-69.

19. Giganchi P, Estambale B, Bwayo J, Rogo K, Ojwang S, Opiyos A, et al. Knowledge and practice about cervical cancer and Pap smear testing among patients at Kenyatta National Hospital, Nairobi, Kenya. Int J Gynecol Cancer. 2003;13:827-33.

20. Klug S, Hetzer M, Blettner M. Screening for breast and cervical cancer in a large German city: participation, motivation and knowledge of risk factors. European Journal of Public Health. 2005;15(1):70-7.

21. Lazcano-Ponce E, Moss S, Cruz-Valdez A, De Ruiz P, Casares-Queralt S, Martinez-Leon C, et al. Factores que determinan la participación en el tamizaje de cáncer cervical en el estado de Morelos. Salud Pública de México. 1999;41(4):278-85.

22. Aguilar-Perez J, Leyva-López A, Angulo-Nájera D, Salinas A, Lazcano-Ponce E. Tamizaje en cáncer cervical: conocimiento de la utilidad y uso de citología cervical en México. 2003;37(1):100-6.

23. Valenzuela M, Miranda A, ¿Por qué NO me hago el Papanicolau? Barreras sicológicas de mujeres de sectores populares de Santiago de Chile. Rev Chil Salud Pública. 2001;5(2):75-80.

Manuscrito recibido el 13 de febrero de 2007 y aceptado para publicación el 05 de marzo de 2007.

Correspondencia:

Fernando Bazán Asencios

Av. Libertad 2096, Urb. Maranga.

Lima 32, Perú

Correo-e:fer_bazan@hotmail.com 